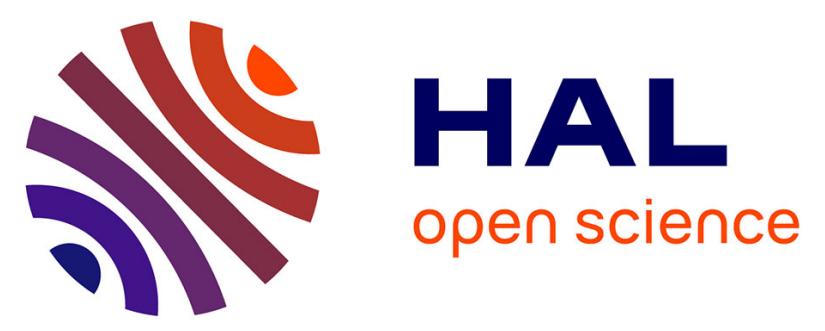

\title{
Automatic Optimisation of Reliable Collaborative Services in OLSR Mobile Ad Hoc Networks
}

Christophe Guettier, Philippe Jacquet, Laurent Viennot, Jacques Yelloz

\section{To cite this version:}

Christophe Guettier, Philippe Jacquet, Laurent Viennot, Jacques Yelloz. Automatic Optimisation of Reliable Collaborative Services in OLSR Mobile Ad Hoc Networks. Proceedings of IEEE MILCOM: Military Commununications Conference, Oct 2007, Orlando, United States. pp.1-7, 10.1109/MILCOM.2007.4454993 . inria-00471721

\section{HAL Id: inria-00471721 \\ https://hal.inria.fr/inria-00471721}

Submitted on 8 Apr 2010

HAL is a multi-disciplinary open access archive for the deposit and dissemination of scientific research documents, whether they are published or not. The documents may come from teaching and research institutions in France or abroad, or from public or private research centers.
L'archive ouverte pluridisciplinaire HAL, est destinée au dépôt et à la diffusion de documents scientifiques de niveau recherche, publiés ou non, émanant des établissements d'enseignement et de recherche français ou étrangers, des laboratoires publics ou privés. 


\title{
Automatic Optimisation of Reliable Collaborative Services in OLSR Mobile Ad Hoc Networks
}

\author{
Christophe Guettier (1), Philippe Jacquet (2), Laurent Viennot (1), Jacques Yelloz (2) \\ (1) SAGEM DS, 178, Rue de Paris 91300 Massy, France \\ (2) INRIA, Rocquencourt B.P. 105, France
}

\begin{abstract}
-
Future Network based Operations (NbO) will strongly rely on Mobile Ad hoc Networks (MANET), due to urban area, tactical mobility and assymetric operation management. These networks will support multiple collaborative services, such as sensor to shooters, reachback, maintenance of Common Operational Picture (COP). Both networks and services will have to be managed with no or limited operator intervention, still providing reliable behavior in aggressive environments. At routing level, we present how to preserve 2-connectivity by adapting the Optimised Link State Routing Protocol (OLSR). We make use of the specific parameter MPR-COVERAGE in the Multiple Relay Selection algorithm in order to conserve 2-connectivity. At the service level, the operational gain grows with the numbers of actors, as they can bring multiple skills and data in a given collaborative activity. Following a constraint solving method, the paper shows how to maximise a subnet of actors involved in a given collaborative service, while satisfying 2-connectivity, reactivity and communication quality constraints. We demonstrate the approach on simulating real world $\mathrm{NbO}$.
\end{abstract}

\section{INTRODUCTION}

The future concept of Network based Operations $(\mathrm{NbO})$ is a new approach to deal with assymetric context, urban area and tactical mobility. In $\mathrm{NbO}$, critical decisions can be optimised thanks to a better information sharing between commanders. Examples can be found in operational pictures, mission planning and execution. $\mathrm{NbO}$ also opens new spectrum of capabilities (short loops between key-players, experts reachback, remote navigation of drones and robots, ...) based on communication and information advantage. These new collaborative activities must be supported by reliable distributed services, subject to performance guarantees.

Over the last fifty years, significant advances have led to the development of high-performance deploybale defence network infrastructure. Finding many essential applications in both defense and civilian domains, these network systems are based on high gain fixed antenae coupled with high- performance routing devices. However, most often, these systems rest on complex and rigid deployment, which results in little mobility and reconfiguration capabilities.

Opposed to these concepts, a new generation of mobile ad hoc networks (MANET) is trading off high-performance with availability, mobility, flexibility, affordability and survivability. Although these mobile ad hoc networks are inheriting advances in physical layers, they must be rapidly deployed into a non-cooperative (not to say aggressive) environment, for which an incomplete or uncertain knowledge is available. These requirements are stressed in modern assymetric operations, due to urban area, tactical mobility and uncertain environment where deployed infrastructure cannot be easily protected. Subject to link break, jamming, node failures and message losses, these networks must constantly optimise their own organisation in order to deliver reliable services.

In addition, service management levels must deal with service demands, environment changes or mission contingencies. Admiting services and actors to support collaborative activities becomes difficult since these decisions must satisfy performance, robustness and feasibility constraints - also referred to as Quality-of-Service (QoS).

This paper proposes an approach based on satisfying the reliability property, locally at the routing level as well as globally at the service one. Locally, we demonstrate how to preserve network 2-connectivity, wherever it does not exhibit single point of failure (network edge or node). Globally, we show how to map a collaborative service on an optimised subnet topology which also guarantee 2 -connectivity.

Research and applied works have already considered the Optimised Link State Routing (OLSR) protocol to address mobile tactical communications, as it saves topology control messages and provides proactively a route always available between any two nodes. We make use of the specific parameter MPR-COVERAGE in the Multiple Relay Selection algorithm for OLSR in order to conserve 2-connectivity and to dynamically detect such topologies. This strong property can be proven and does not overload significantly the OLSR protocol in terms of control messages nor protocol complexity.

At the service level, the operational gain grows with the 
number of actors, as they can bring multiple skills and data in a given collaborative activity. However a large set of interacting actors may involve too much traffics or behave too slowly. The problem is to configure automatically a maximal subset of actors (or subnet) involved in a collaborative service, while satisfying end-to-end reactivity as well as reliability constraints. Compliant with the routing level, subnet reliability is assimilated as a 2-connected topology. We show that under specific assumptions, this problem corresponds to a maximal clique problem, well known to be NP-hard. To tackle its complexity, it is first modeled as a constrained optimisation problem and solved using a combination of branchand-boud, constraint propagation techniques, mathematical inference and greedy anytime heuristics.

We demonstrate the approach on simulating real world $\mathrm{NbO}$ using both constraint solving programming and discrete event-based simulation of OLSR ad hoc network. Experiments, focused on tactical data, show that optimal collaborative services can be found in reasonable time, in line with the operational tempo. These first return on experiments illustrates a new way to perform late binding between ad hoc networks and collaborative services in spite of reactivity constraints and high disponibility requirements.

\section{Collaborative SERVICES IN AD HOC NETWORKS}

\section{A. Background}

The new defence concept Network based Operation (NbO) enables decision superiority thanks to a better information sharing between commanders. This is a promising approach in terms of operational gain such as accelerating the peace of events, optimising resources and minimizing frictions. $\mathrm{NbO}$ will strongly rely on collaborative and critical services, such as sensor to shooters, reachback, maintenance of common operational picture, mission planning and execution. Network nodes can be different actors such as vehicles, soldiers, sensors, UAV or UGV, such as pictured in figure (1). They will generate many and permanent traffics as well as different scales of mobility. With these heterogeneous actors, collaborative activities will require frequent services reconfiguration due to mission contingencies and uptades, topology or environment changes. Networks and services will have to be managed automatically, with no or limited operator intervention, and must guarantee correct behaviours, end-to-end performances in spite of aggressive environments. The difficulty to simultaneously operate service management and perform efficient routing in a reliable way is challenging the design of modern tactical defence networks.

A representative example of a service supported by a MANET is the Common Operational Picture (COP). Within the network, a subnet of "available" actors cooperate to exchange tactical data. The service must support the delivery of

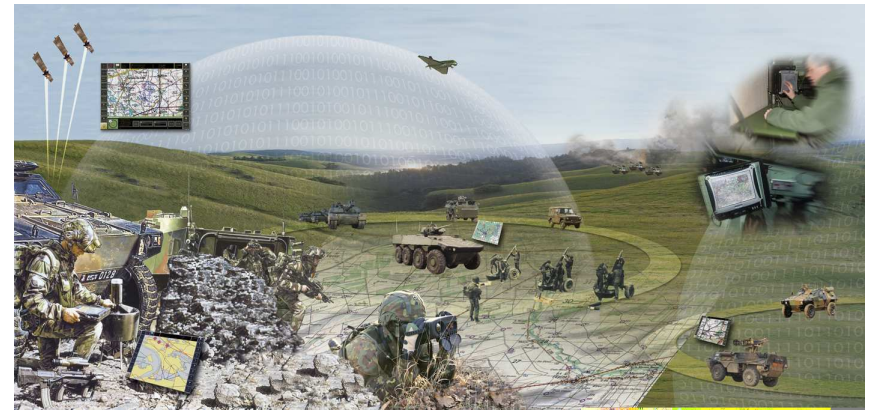

Fig. 1. Actors of an operation potentially participating to a collaborative service

tactical information by any subnet actor to every other subnet actor (for example unit detection, identification, ... ). The aim is that all actors in the subnet consolidate the COP, within a specified end-to-end latency.

When the environment is changing, the subnet must decide which new actors shall join the subnet and which existing subnet members shall leave, in order to maximize information quality or freshness. The set of actors composing the new subnet must be choosen so that it is fully connected, and it satisfies the different criteria relevant to Quality of Service:

- The level of reliability is acceptable;

- End-to-end latency must be lower than a specified bound;

- Message / processing loads are met.

\section{B. Active Subnet Management}

To consolidate efficiently the COP, the subnet must continuously deliver the required information in spite of two kinds of environment changes:

1) Environment change status: status are broadcasted through the subnet with other tracking information.

2) Routers fail or appear in the environment. Network topology is broadcasted throughout the network.

Testing each sensor router one by one is not a satisfactory approach, because the different criteria involve both members of the existing subnet and the set of new actors. Such "generate and test" approach has to evaluate simultaneously all the criteria for any subset of the total set of available routers (including existing subnet members and new routers), which corresponds to $2^{N}$ combinations. In order to find a feasible solution and to potentially maximize the amount of information retrieved in the COP, a "generate and test" approach can not be really applied and it is necessary to solve globally the subnet management problem, considering the different criteria as hard constraints.

This constrained optimization problem can be illustrated with figure 2. Supposing that active subnet members $\{S 1, S 2, S 3, S 4\}$ do not provide enough quality information about targets $T 2$ and $T 3$, any subset of $\{N 1, N 2, N 3, N 4\}$ 
would improve COP performance. Supposing, furthermore, that admitting $\{N 3, N 2\}$ would provide more information, too much communication would be generated (since both sensors would communicate data about $T 3$ and $T 4$, the new subnet might be saturated by the new traffic), or the COP process would be too slow. Admitting $\{N 1, N 3\}$ or symmetrically $\{N 4, N 2\}$, on the other hand, would increase significantly information quality about $T 2$ and $T 3$ and would generate an acceptable level of communication, provided $S 1$ and $S 2$ remain in the subnet with their existing links. Also, $S 3$ and $S 4$ cannot leave the network as they maintain connectivity for relaying informations on $\{T 1, T 2, T 3\}$.

The question is, how is this solution derived, given such constraints as broadcast latency and reliability? This is a constrained optimisation problem crucial to providing QoS. However, the problem is interesting to solve only if the routing protocol guaratee same latency and reliability constraints.

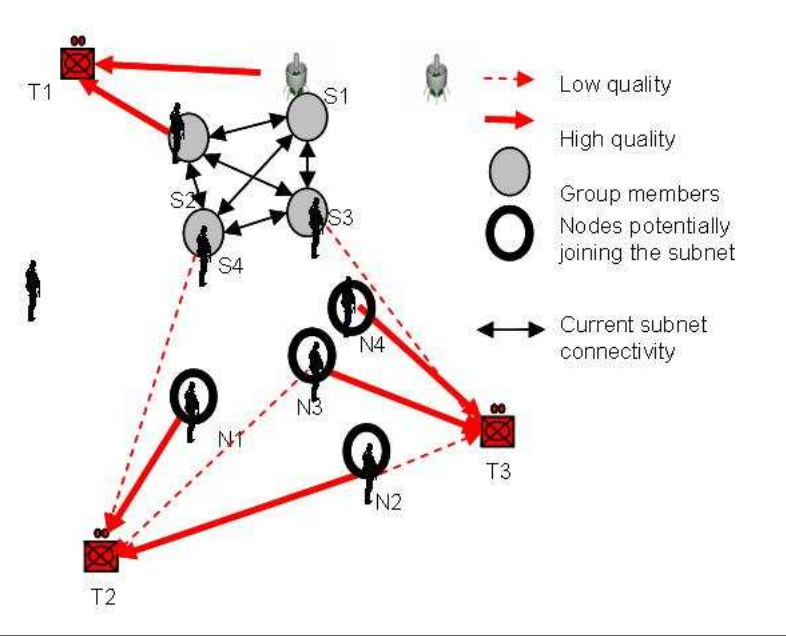

Fig. 2. Distributed tracking with ad-hoc networks

\section{Decision making criteria}

One of the criteria required for admiting a set of actors within the subnet is the measurement of information gain. Other criteria, later considered as hard constraints for admiting a set of actors within the subnet, are essential to provide QoS:

- Reliability The subnet must continue to operate in spite of any node or link immediate stop.

- Latency This very important criterion depends on the link delays and the number of hops on a given route. Overheads due to topology control and reliability mechanisms must also be included when modelling the latency criteria.

Indeed, these criteria are relevant to the Quality of Service only if the routing layer also supports similar reliability and latency constraints.

\section{RELIABLE ROUTING IN OLSR}

\section{A. Network modelling}

Let assume that the network is deployed on a square map of area $\mathcal{A}$. Let $N$ be the total number of nodes in the network and $M$ be the average number of neighbor nodes per node. The average number of hop, assuming all pair of source and destination are equally likely, is exactly $\frac{\ell}{\sqrt{\mathcal{A}}} \sqrt{\pi} \sqrt{\frac{N}{M}}$. Quantity $\ell$ refers to the average euclidian distance between nodes in a square, i.e. $\ell \approx 0.3826 \sqrt{\mathcal{A}}$. The average maximum number of hops, attained in the diagonal is equal to:

$$
H_{\max }=\sqrt{2 \pi} \sqrt{\frac{N}{M}}
$$

This is not an integer number because there are variations due to node positioning.

Gupta and Kumar have shown that the network stays connected as long as $M<\log N$ which gives an average maximum number of hops equal to $H_{\max }=\sqrt{2 \pi} \sqrt{\frac{N}{\log N}}$.

With $N=30$ the maximum number of hops will be in average 7.4444 , and in average nodes are separated by 2.014 hops.

\section{B. Link quality modelling}

Assume $\lambda$ is the density of emitter per slot and per heactar, assuming each emitted power is of one unit, assume also that the minimum Signal-over-Noise Ratio (SNR) $K>1$, then the probability that a packet is correctly received at distance $r$ is $p\left(r \frac{\sqrt{\lambda}}{K^{\frac{1}{\alpha}}}\right)$ where $\alpha$ is the signal attenuation factor and with

$$
p(x)=\sum_{N \geq 0}\left(\Gamma\left(1-\frac{1}{\alpha}\right)\right)^{N} \frac{\sin \left(\frac{\pi N}{\alpha}\right)}{\pi} \Gamma\left(\frac{N}{\alpha}\right) x^{2 N}
$$

Euler function $\Gamma(s)=\int_{0}^{\infty} e^{-x} x^{s-1} d x$ is defined for $R e(s)>0$ and extended to all $\mathrm{s}$ in the complex plan excepted the negative integer: $\Gamma(1+s)=s \Gamma(s)$ and $\Gamma(n)=(n-1)$ ! for every n natural integer.

Assuming a random transmission with collision detection, the average number of packet retransmission over a distance $L$ is $\frac{L}{r p\left(r \frac{\sqrt{\lambda}}{K^{\frac{1}{\alpha}}}\right)}$, assuming that two consecutive relays are at most distance $r$ apart. The hop radius $r$ that minimizes this quantity is $r_{0} \frac{K^{\frac{1}{\alpha}}}{\sqrt{\lambda}}$ with $r_{0}=\arg \max \{r p(r)\}$. For $K=4$ and $\alpha=4$ we got a radius $\sqrt{\frac{0.181}{\pi \lambda}}$ and $p_{0}=p\left(r_{0}\right)=0.653$.

The radius can be tuned if other QoS metric or link cost is considered.

\section{OLSR modelling}

Let $\rho$ be the traffic load per node. This traffic load is made of (i) neighbor sensing traffic $\rho_{v}$, (ii) the topology dissemination load $\rho_{t}$, and (iii) the useful payload traffic $\rho_{d}$. We have 
$\rho=\rho_{v}+\rho_{t}+\rho_{d}$. We have $\rho_{v}=h\left\lceil\frac{M}{B}\right\rceil$ where $h$ is the hello frequency per slot, $B$ is the slot capacity (in number of node's ID in hello packets). We also have $\rho_{t}=\tau\left\lceil\frac{M_{r}}{B}\right\rceil \frac{M_{r}}{M} N$ where $\tau$ is the frequency of per node TC message generation (e.g. $h=1 / 1000, \tau=1 / 5000$ with 1000 slots per second). Quantity $M_{r}$ is the average number of multipoint relay selected by a random node. With MPR-coverage at 2 it is approximately $5 M^{\frac{1}{3}}$. We have $\rho_{d}=\frac{\mu}{p_{0} \mu_{0}} \sqrt{\frac{N}{M}}$ with $\mu$ as the average net payload generated by a node (excluding mode relaying) and $\mu_{0}=\frac{\sqrt{\mathcal{A}}}{\ell \pi}$. The following equilibrium equation holds as long as $M>\log N$ :

$$
\frac{\sigma_{0}}{M}=\rho_{v}+\rho_{t}+\rho_{d}
$$

This equation holds for some $M>N$ in this case the network is not loaded enough to split in multihop and every pair of nodes is connected. This analysis does not take into consideration the presence of permanent obstacles between nodes.

\section{Preserving 2-connectivity with OLSR}

A graph is 2-connected when it is connected and remains connected after removal of a vertex. Equivalently, every pair of nodes is connected by two node disjoint paths. This property ensures reliability towards node failure. However, link state ad hoc routing protocols such as OLSR rely on a reduced subset of the topology in order to optimize control traffic. Such optimization may lead to route computations on a graph that is not 2-connected even in a 2-connected network. A node failure may then cause traffic loss until new sets of advertised links are computed and disseminated. On the other hand, ensuring 2-connectivity of route computations allows to maintain a backup route for each destination and use it as soon as a link breakage is detected.

OLSR uses multipoint relays (MPR for short) [?] both for optimizing flooding of control packets and for selecting a subset of advertised links. Each node selects MPRs among its neighbors so that any 2-hop neighbor is covered by at least one MPR. This property ensures that flooding through MPRs reach all nodes. Moreover, it allows to show that MPR links (links from a node to one of its MPR) form a sub-topology sufficient to compute shortest routes.

A simple greedy algorithm allows to find MPR sets with small size [?]. The algorithm can be tuned to allow better coverage of 2-hop neighbors through the MPR-COVERAGE parameter $k$ [?]. This parameter specifies that each 2-hop neighbor should be covered by at least $k$ MPRs. More precisely a 2-hop neighbor covered by $i$ neighbors must be covered by $\min (i, k)$ MPRs. This parameter allows to introduce more redundancy in flooding. It has been shown recently [?] that it also ensures a $k$-connectivity property of the MPR links. Namely, any node $u$, using knowledge of its neighbors and MPR links can compute $k^{\prime} \leq k$ node disjoint routes to each destination as long as there exists at least $k^{\prime}$ node disjoint paths in the network.

A weaker reliability property is edge 2-connectivity: a graph is edge 2-connected if it is connected and remains connected after removal of an edge. Equivalently there exists 2 edge disjoint paths between any pair of nodes. Notice that node disjoint paths are edge disjoint. But, it may happen that $k^{\prime}$ edge disjoint paths exist between two nodes when less than $k^{\prime}$ node disjoint paths can be found. However, it is shown [?] that MPR-COVERAGE $k \geq k^{\prime}$ also preserves this property. Namely, an OLSR node can compute $k^{\prime}$ edge disjoint routes as long as such routes exist and all MPR sets are computed with MPR-COVERAGE $k \geq k^{\prime}$.

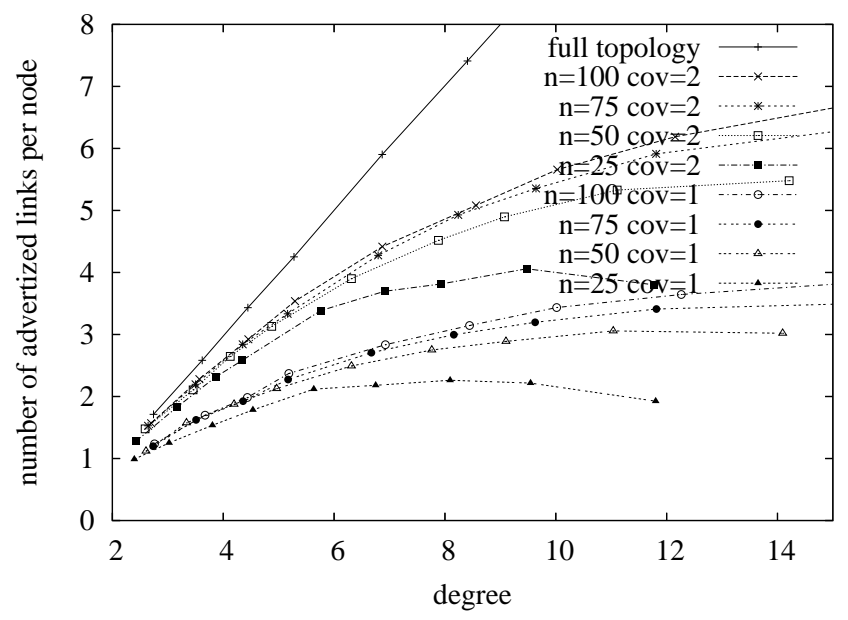

Fig. 3. Average number of MPRs versus average degree for various number of nodes $n=25,50,75,100$ and MPR-COVERAGE $k=$ $1,2$.

Figure 3 illustrates the overhead of traffic control for various size and density of networks with MPR-COVERAGE 1 or 2. Indeed, each TC contains the list of MPR selectors of a node. The average size of a TC is thus proportional to average number of MPRs of a node. We can see that switching from MPR-COVERAGE 1 to 2 almost doubles the size of topology control messages (TCs). As MPR flooding will also require roughly twice more emissions, the traffic control is thus roughly multiplied by a factor 4 . Notice that it could be possible to compute TCs with MPR-COVERAGE 2 when flooding according to MPR-COVERAGE 1 to impact traffic control by a factor less than 2 .

\section{Modelling And Solving the Maximal Reliable Subnet Problem}

Constraint Programming (CP) has been shown to be an efficient framework for designing solving algorithms that can cope with large scale combinatorial problems. In the approach, CP is used to formulate constraint based models as 
well as to design efficient search strategies. The algorithm combines shortest path with anytime search technique.

Both problem model and search algorithm are developed and experimented in SICSTUS Prolog that provides a constraint solving library based on AC4 and AC5 as well as facilities to design incremental search techniques.

\section{A. Constraint-based modelling}

Model-based constraint solving exploits different representations of a problem structure. The modelling process starts by capturing the problem invariants, and refines this using several approximations, sufficient conditions and abstractions. A model consists of constraints and mathematical variables that represent partially or totally the problem to be solved. In our context, a model-based approach is required to formulate and compose the different QoS constraints.

Various options can be envisaged to model the subnet management problem. For illustrating our approach, we give the main constraints that model subnet problem, with information quality as a cost function. Radius and reliability constraints are also modeled. Bandwidth capacity constraints are much more difficult to model in details and we must rely on OLSR extensions to guarantee available bandwidth. A rough approximation can be done by limiting the number of subnet members, based on their bandwidth utilisation and equation (3).

Decision variables $X_{p} \in\{0,1\}, 0 \leq p<N$, model the set of sensor routers that may be admitted in a subnet. When $X_{p}$ is positive, router $p$ is considered active subnet member.

1) Radius constraints: The first constraint limits the number of hops between any two subnet nodes. This limit results from constant $H_{\max }(1)$ and (2), and can both be preprocessed in polynomial time.

The distance between every pair of active subnet members does not exceed constant $h_{\max } \in \mathbb{R}$ (where distances $d\left(p, p^{\prime}\right) \in \mathbb{R}$ between each pair of sensor routers $\left(p, p^{\prime}\right)$ are considered constant and known at problem solving time) (4):

$$
\forall p, p^{\prime}, \min \left(X_{p}, X_{p^{\prime}}\right) \cdot d\left(p, p^{\prime}\right)<h_{\max }
$$

2) Reliability: Reliability constraints derive from Menger's theorem and is more intricate to state. The aim of these constraints is too represent a node-disjoint path between any two pair of nodes belonging to the subnet (Fig. 4.

The network topology is represented as a graph $G(X, U)$ where the set of edges $U$ is representing communication links, the set of vertices $X$ admissible nodes ${ }^{1}$, and indice $p / p \in$ $\{0, \ldots n-1\}$ denotes a given node. For each couple of nodes $\left(p, p^{\prime}\right)$, two sets of variables respectively $\left\{\varphi_{u}^{p p^{\prime}}\right\}_{0}^{U-1} \in\{0,1\}$ and $\left\{\hat{\varphi}_{u}^{p p^{\prime}}\right\}_{0}^{U-1} \in\{0,1\}$ models a couple of disjoint path

\footnotetext{
${ }^{1}$ In the remaining of the paper, a vertex is denoted $x$, while an edge can be denoted either $u$ or $\left(x, x^{\prime}\right)$, a possible subnet member $X_{p}$
}

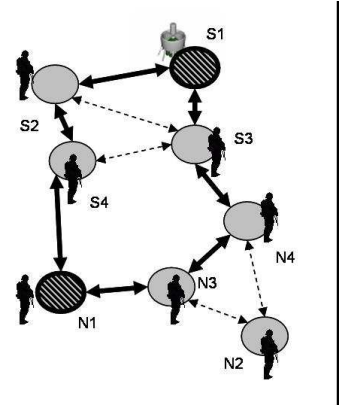

This figure illustrates a couple of node disjoint paths. Their existence is guaranteed by tuning OLSR protocol with MPR-COVERAGE parameter, and is imposed within the subnet by satisfying constraints (9).

Fig. 4. Node disjoint routes (in bold) between two nodes.

between $p$ and $p^{\prime}$. An edge $u$ belongs to the primary path $\Phi$ (resp. secondary $\hat{\Phi}$ ) path iff variable $\varphi_{u}^{p p^{\prime}}$ (resp. $\varphi_{u}^{\prime}$ ) is instantiated to $1(5,6)$.

$$
\begin{array}{r}
\forall p \in X, \forall p^{\prime} \in X / p<p^{\prime}, \\
\Phi^{p p^{\prime}}=\left\{\varphi_{u}^{p p^{\prime}} \mid u \in U, \varphi_{u}^{p p^{\prime}}=1\right\} \\
\hat{\Phi}^{p p^{\prime}}=\left\{\hat{\varphi}_{u}^{p p^{\prime}} \mid u \in U, \hat{\varphi}_{u}^{p p^{\prime}}=1\right\}
\end{array}
$$

From an initial node $p$ to a final node $p^{\prime}$, path consistency is asserted by the following constraints, where $\omega^{+}(x) \subset U$ and $\omega^{-}(x) \subset U$ are outgoing and incoming edges from vertex $x$, respectively.

$$
\begin{array}{r}
\forall p \in X, \forall p^{\prime} \in X / p<p^{\prime}, \\
\sum_{u \in \omega^{+}(p)} \varphi_{u}^{p p^{\prime}}=X_{p}, \sum_{u \in \omega^{-}\left(p^{\prime}\right)} \varphi_{u}^{p p^{\prime}}=X_{p^{\prime}} \\
\forall x \in X \backslash\left\{p, p^{\prime}\right\} \\
\sum_{u \in \omega^{+}(x)} \varphi_{u}^{p p^{\prime}}=\sum_{u \in \omega^{-}(x)} \varphi_{u}^{p p^{\prime}} \leq 1,
\end{array}
$$

Equation (8) ensures path connectivity and unicity while equation (7) imposes limit conditions for the extremities of the path. Note that a path cannot be built between any node outside and any node inside the subnet.

A similar constraint is also stated for $\hat{\Phi}$. Then, the node disjoint constraint (9) can be stated between the two path and any two nodes of the network (Fig. 4):

$$
\forall x \in X \backslash\left\{p, p^{\prime}\right\}, \quad \sum_{u \in \omega+(x)} \varphi_{u}^{p p^{\prime}}+\sum_{u \in \omega+(x)} \hat{\varphi}_{u}^{p p^{\prime}} \leq 1
$$

3) Maximizing information quality: Finally, the global gain $G$ of the network is maximized using the following linear function:

$$
G=\sum_{p=0}^{N-1} g_{p} \cdot X_{p}
$$

where constant $g_{p}$ represents information quality introduced by node $p$. 


\section{B. Constraint solving}

\section{explain (1) NP complete and (2) why LP is not adapted}

In constraint programming, inference involves propagation algorithms, such as Arc-Consistency (AC). These algorithms perform domain filtering by maintaining feasible assignments (AC4), or by reducing variable domains (AC5), both of which can be performed in polynomial time. However, to solve the subnet management problem, such incomplete methods must be supplemented with other techniques. Arc-consistency algorithms are incomplete since they stop at a fixed point when the set of variables becomes arc-consistent, which does not guarantee that a solution exists in the resulting variables domains. To filter consistent variable assignments, hybrid algorithms are developed.

Therefore, search algorithms have to be designed for completing constraint solving, by exploring consistent or inconsistent assignment of variables. Using a CP framework, solving methods consist in filtering consistent assignments of the set of variables, by exploring search trees. Optimization schema, such as branch and bound or iterative deepening are also combined with the solving method.

In addition, these search algorithms must operate in a dynamic environment, where part of the solution already exists and has to be adapted to a new input. This is required when adapting the subnet to environment changes and traffic demand. Anytime search methods can provide a solution of increasing quality. Examples of such methods are:

- Probe Backtracking (PB)[?], which repairs a tentative assignment to the set of variables..

- Repair Algorithms (RA) [?] [?], which approximate incrementally feasible variable assignments by minimizing the degree of inconsistency.

- Valued Constraint Satisfaction Problems (VCSP), which associate a priority to each constraint model. Constraints are satisfied in an order compliant with priorities.

Such algorithms proceed incrementally and can be interrupted at any time, providing a solution of a given quality. This approach is likely to provide the requisite flexibility for subnet management to be executed in a dynamic environment.

The constraint solving algorithm in use instantiate variables $X_{p}$. The algorithm starts by a single node. Then, candidate nodes are added incrementally by exploring all possible assignement to variables $X_{p}$. These assignements are always satisfying subnet constraints (4,7,8 and 9). A branch and bound loop iterates over the search algorithm to maximise the cost function (10).

This way, the solving algorithm can be interupted a anytime, providing an optimised solution, consistent with subnet model constraints.

\section{EXPERIMENTATIONS}

\section{A. Analysis and experimentation environment}

The experimentation environment is coupling an OLSR simulator with the reliable subnet optimisation algorithm described in $\oint$ IV-A. The environment takes as input a network based operation scenario and generates QoS measurements. In particular, the tool can report when equilibrum (3) is broken. The simulation activates the optimisation whenever the scenario requires it. In turn, the simulator manages sensing, topology dissemination and payload traffics related to the subnet.

The simulator is based on a discrete event representation and focuses on modelling OLSR routing mechanisms. It uses the MPR-COVERAGE parameter to conserve 2-connectivity. Units mobility are also modelled using realistic data and mission plans. This impacts dynamically network topology and related OLSR traffics.

\section{B. Example scenario}

The scenario takes place in a town where some check points must be visited or controlled by a company (or squadron). During the mission, collaborative services are set to coordinate the different units (for example, some units must control a cross street while other are visiting a check point). The simulator generates dynamically payload traffics according to service activity and related actors. The optimised subnet is then constructed to support activation of the collaborative service. In figure 5, the optimal subnet with a maximum of 9 members is represented using the black dots, and subnet payload traffics by bold lines.

\section{Results}

Experimentations vary the maximal number of subnet members, among a set of 16 nodes, operating in a square of $10 \times 10 \mathrm{kms}$. Within a subnet, a multicast payload traffic of $1 / 10$ of the maximal available bandwidth is generated. The topology is composed with 120 edges. This scenario instance represents 65552 variables and 17162 constraints.

Figure (6) shows processing time (y-axis) for computing an optimal subnet with a maximum of 8 members. In practice, results exhibit linear solving time, which confirm the anytime behavior of the search algorithm. Due to network density, the $h_{\text {max }}$ does not impact significantly performances, since 2-connected subnet can be easily found.

However, these orders of magnitude on a realistic scenario demonstrate the concept feasibility at the company (or squadron) level.

Figure (7) shows subnet performances with OLSR and MPR-coverage set to 2, in terms of latency (y-axis, on the left hand side) and packet delivery (y-axis, on the right hand side) according to the maximal number of subnet members 


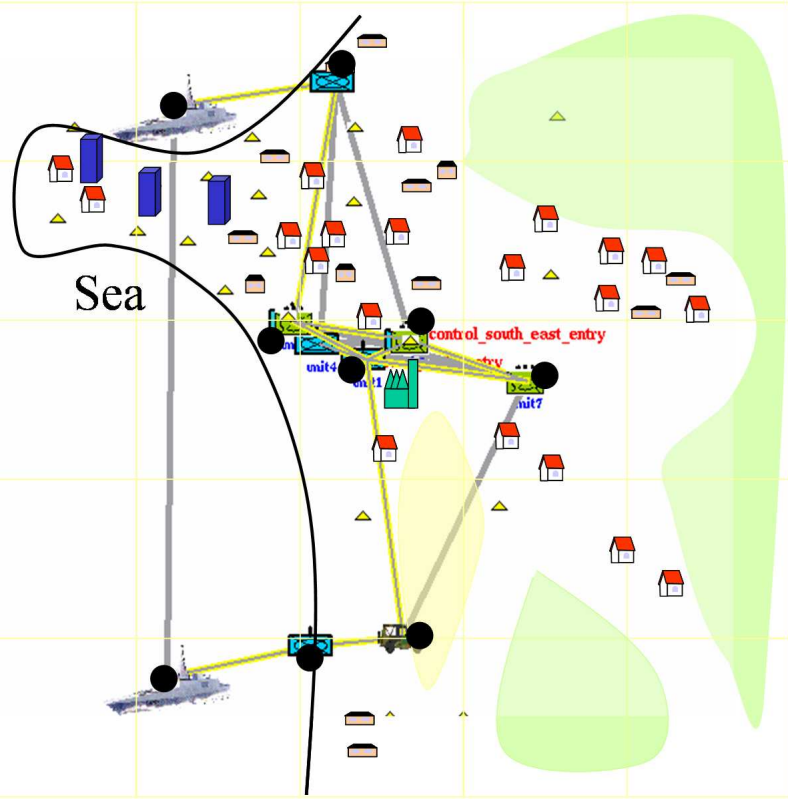

Fig. 5. Optimal reliable subnet for a collaborative service during the simultaneous visit and control of check points in a peace keeping mission.

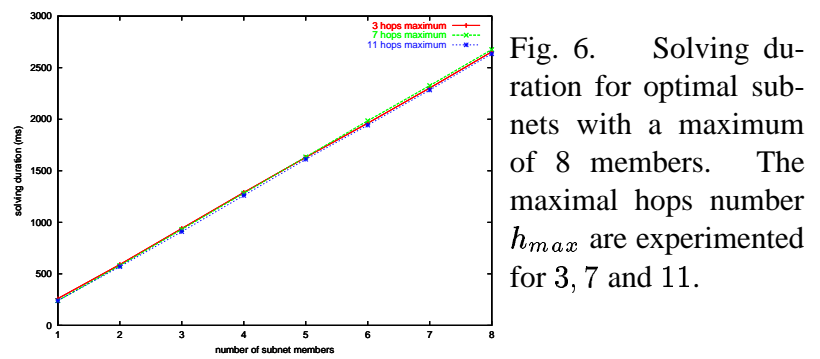

(x-axis), among the 16 nodes. These experiments are performed with a maximum hop number $h_{\max }$ set to 7 . The performance sample is selected on a time window which starts at subnet activation.

The curves exhibit a breaking point where the equilibrum (3) is broken, due to saturated edges. On this scenario instance, this reveals both OLSR and subnet model stability with nominal load conditions in terms of quality of service.

\section{CONCLUSION}

\section{consensus + overload}

\section{ACKNOWLEDGEMENT}

We thank Prof. Barry Richards for his constructive comments and his strong support writing this paper.
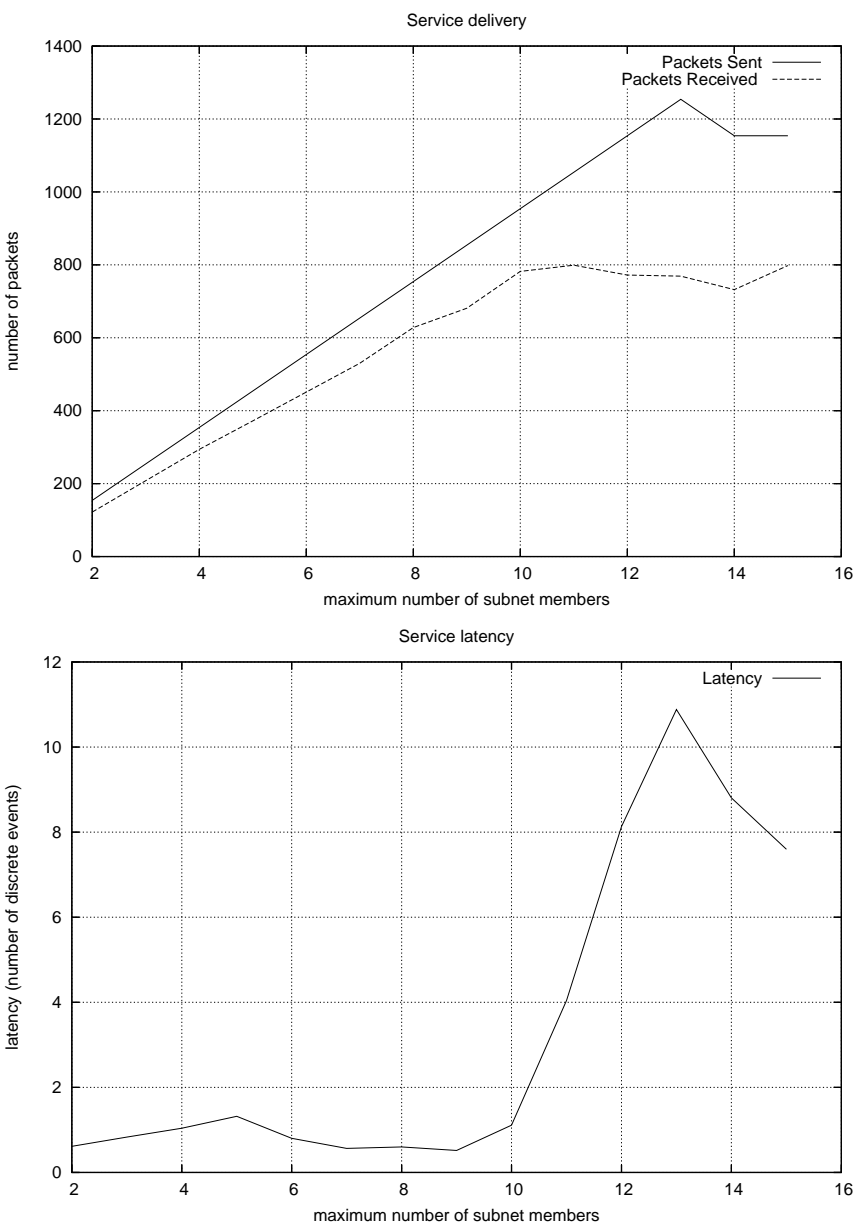

Fig. 7. Latency and packet delivery according to subnet maximal sizes 